\title{
Neues Materialkonzept für Solarzellen
}

\author{
Ein grundlegend neues Solarzellenkonzept wollen Wissenschaftler des Karlsruher Instituts für \\ Technologie (KIT) im Projekt „Neuartige flüssig-applizierte keramische Solarzellen“ (KeraSolar) \\ entwickeln. Dabei verbinden sie Ansätze zu Photovoltaik mit keramischen Funktionsmateria- \\ lien, um so die Vorteile verschiedener Solarzellentechnologien zu bündeln: Die Druckbarkeit \\ organischer und die Langzeitstabilität kristalliner Solarzellen sowie die Ferroelektrizität des \\ Bleihalogenid-Perowskits.
}

Einer der wichtigsten Grundpfeiler der künftigen $\mathrm{CO}_{2}$-neutralen Energieversorgung ist die Sonnenenergie. Solarzellen können diese einsammeln und in nutzbare elektrische Energie umwandeln (Bild 1). In den nächsten sechs Jahren beschäftigen sich Forscher des KIT in dem von der CarlZeiss-Stiftung mit 4,5 Millionen Euro geförderten Projekt "Neuartige flüssigapplizierte keramische Solarzellen" (KeraSolar) mit einem völlig neuen Materialkonzept für Solarzellen.

Die neuen Funktionsmaterialien werden aus Keramikwerkstoffen hergestellt, die eine außerordentlich gute Robustheit und Langzeitbeständigkeit versprechen. Moderne Solarzellen müssen aber weit mehr Eigenschaften besitzen: Sie müssen beliebig formbar und integrierbar sein, um so quasi jede Fläche in Sonnenkraftwerke zu verwandeln. Ihre Produktion muss so wenig Energie wie möglich verbrauchen, die Herstellungsprozesse sollten ohne giftige Substanzen auskommen und die notwendigen Rohstoffe ausreichend verfügbar sein. Genau hier kommen die Vorteile keramischer Funktionsmaterialien zum Tragen: Sie bieten nahezu unendliche Möglichkeiten, Ele- mente und Verbindungen miteinander zu kombinieren und so maßgeschneiderte Materialeigenschaften zu erzielen. Damit eröffnet sich dem Projektteam ein großes neues Forschungsfeld.

Das Projekt ist am Materialwissenschaftlichen Zentrum für Energiesysteme (MZE) des KIT angesiedelt, dessen Programmatik sich an den großen Forschungsthemen rund um die Energiewandlung und -speicherung orientiert. „Wir freuen uns, dass sich das MZE innerhalb kürzester Zeit zu einem Vorzeigeprojekt der modernen, multidisziplinären Materialforschung entwickelt hat, dessen Leistungen durch KeraSolar anerkannt und nachhaltig unterstützt werden", sagt Professor Michael J. Hoffmann, einer der Initiatoren des MZE. „Wir freuen uns riesig über die sechsjährige Förderung durch die Carl-Zeiss-Stiftung."

Etwa die Hälfte der 16 am MZE angesiedelten Arbeitsgruppen aus unterschiedlichen wissenschaftlichen Disziplinen werden an dem Projekt mitwirken und ihre Expertise aus der Elektrotechnik, den Materialwissenschaften, der Physik und der Chemie einbringen. Sie verbinden experimentelle Herangehensweisen mit theoretischen

\section{Über die Carl-Zeiss-Stiftung}

Die Carl-Zeiss-Stiftung hat sich zum Ziel gesetzt, Freiräume für wissenschaftliche Durchbrüche zu schaffen. Als Partner exzellenter Wissenschaft unterstützt sie sowohl Grundlagenforschung als auch anwendungsorientierte Forschung und Lehre in den MINTFachbereichen (Mathematik, Informatik, Naturwissenschaften und Technik). 1889 von dem Physiker und Mathematiker Ernst Abbe gegründet, ist die Carl-Zeiss-Stiftung die älteste private wissenschaftsfördernde Stiftung in Deutschland. Sie ist alleinige Eigentümerin der Carl Zeiss AG und Schott AG. Ihre Projekte werden aus den Dividendenausschüttungen der beiden Stiftungsunternehmen finanziert.

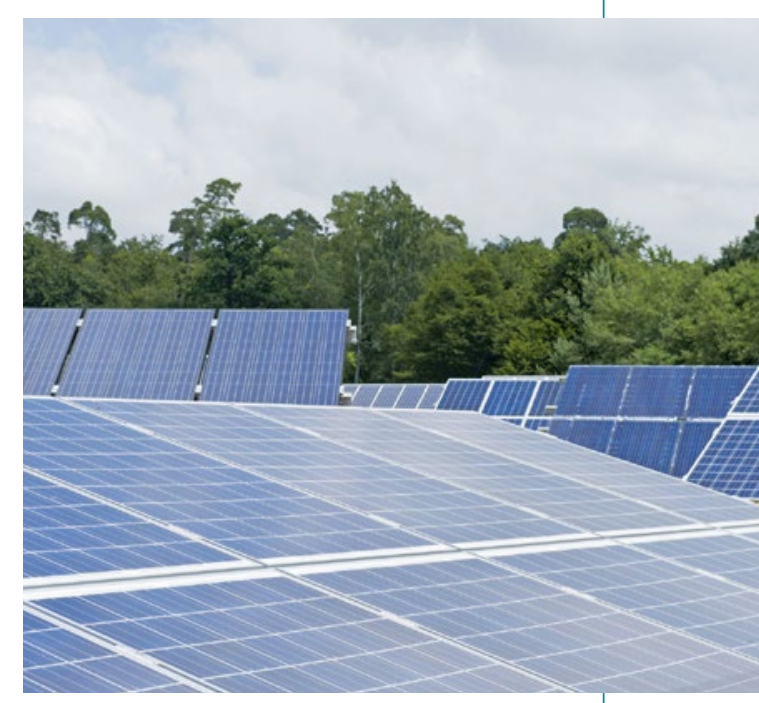

Bild 1 Im Projekt „KeraSolar" des Materialwissenschaftlichen Zentrums für Energiesysteme (MZE) des KIT sollen neue Materialkonzepte für Solarzellen erforscht werden. (@ Markus Breig/KIT)

Überlegungen. „Dank der Wissenschaftler aus vollkommen unterschiedlichen Disziplinen und Hintergründen ist es uns möglich, ein derart herausforderndes Forschungsprojekt auf die Beine zu stellen", so Dr. Alexander Colsmann. Gemeinsam mit Michael J. Hoffmann koordiniert er das neue Vorhaben. Eine neue, speziell für das Projektteam von „KeraSolar" eingerichtete experimentelle Plattform soll dabei helfen, die Solarzellenforschung des KIT langfristig zu prägen.

Kontakt: Karlsruher Institut für Technologie, Karlsruhe, www.kit.edu 